\title{
10 Am Ende der Straße: totale Sicherheit
}

Wie das Sicherheitskonzept von automatisierten und vernetzten Fahrsystemen den Straßenraum verändert

Mathias Mitteregger

1. Verkehrssicherheit als treibende Kraft

$1.1 \quad$ Fahrroboter als Schlüssel zur Sicherheit

1.2 Perspektive Verkehrssicherheit: Wer profitiert?

1.3 Ein ganz neuer Weg

2. Straßen: Transport und Lebensraum

2.1 Die Bedeutung des Straßenraums für Urbanität

3. Von Überwachung zu „Social Engineering“

3.1 Das nie perfekte System

3.2 Ein neues Straßenmedium

Literatur

Mathias Mitteregger

TU Wien, future.lab Research Center

mathias.mitteregger@tuwien.ac.at

(C) Der/die Autor(en) 2021

M. Mitteregger et al. (Hrsg.), AVENUE21. Politische und planerische Aspekte der automatisierten Mobilität, https://doi.org/10.1007/978-3-662-63354-0_10 
„A major aspect of media effects and development appears in the case of the road as a means of transportation. Like writing or radio the ,content ' of the road is always another medium or other media, whether pedestrians, equestrians, wagons or cars. Depending on the type of vehicle-medium, the nature of the road-medium alters greatly" (McLuhan 1960, Part III, 15).

\section{VERKEHRSSICHERHEIT ALS TREIBENDE KRAFT}

Präsentationen, mit denen die Entwicklung selbstfahrender Autos beworben wird, beginnen häufig mit etwas, was mit Michel Foucault ein „Theater der Schmerzen“ genannt werden könnte (Foucault 2012: 42). ${ }^{1}$ Während Fotos zerrissene Schulbusse und halbierte Autos zeigen, unterfüttert die vortragende Person den viszeralen Eindruck mit Zahlen: 1,2 Millionen Menschen werden jedes Jahr im Straßenverkehr getötet, was Verkehrsunfälle zur häufigsten Todesursache für 15- bis 29-Jährige weltweit macht (WHO 2015: 2). Automatisierte Fahrsysteme sollen diese Tragödie beenden. Dazu müssen Menschen das Steuer an lernende Algorithmen abgeben, die den menschlichen Fähigkeiten überlegen und niemals müde, abgelenkt oder betrunken wären.

Verkehrssicherheit ist das dominante Argument mit dem - über ökonomische Interessen hinaus - der gesamtgesellschaftliche Mehrwert von Vernetzung und Automatisierung des Verkehrssystems argumentiert wird. Zahlreiche Akzeptanzstudien weltweit belegen die fundamentale Bedeutung der Sicherheit für die Durchsetzung der Technologie als Ganzes und es dürfte schwer ein Politikpapier zu finden sein, in dem dieser Aspekt nicht mehrmals betont wird. Selbst die Unfälle mit Testfahrzeugen und überschätzen Assistenzsystemen von Serienfahrzeugen tun dieser Sichtweise keinen Abbruch.

In diesem Beitrag wird der durch Automatisierung und Vernetzung angetriebenen Aspekt der Verkehrssicherheit aus der Perspektive des Straßenraums - mit all seinen Beteiligten - betrachtet. Es wird argumentiert, dass durch die bereits begonnene Entwicklung eine Kehrtwende vollzogen wird, mit der tatsächlich das Konzept der Verkehrssicherheit „von den Füßen auf den Kopf gestellt" werden könnte. Eine derartige Neuorientierung würde nicht allein Verkehrssicherheit im engeren Sinn betreffen. Im Rahmen dieses Beitrags wird argumentiert, dass dadurch das auf Sichtbarkeit beruhende Prinzip der Öffentlichkeit untergraben und durch eine neue Form eines kuratierten Miteinanders ersetzt werden könnte.

\subsection{FAHRROBOTER ALS SCHLÜSSEL ZUR SICHERHEIT}

Automatisierte und vernetzte Fahrsysteme müssen sicher bzw. sicherer als das Auto heute sein. Dies gilt als Grundvoraussetzung für die breite gesellschaftliche Akzeptanz dieser Technologie (Lazarus et al. 2018). Dieser Zusammenhang geht über kulturelle Grenzen hinaus und wurde

1 Die dramatischste Präsentation dieser Art für mich war „Advancing the AV opportunity“ von Mark R. Rosekind, Chief Safety Officer von ZOOX, auf dem Automated Vehicle Symposium in San Francisco am 12. Juli 2018. Die Präsentation ist nicht online verfügbar. Teile des Inhalts werden in Shladover et al. 2019 wiedergegeben. 
global nachgewiesen. „Sicherheit“ war der meist verwendete Begriff in allen untersuchten Studien, die in einem Literaturreview erfasst wurden (Jing et al. 2020). Damit wird zum einen der aktuelle Zustand fortgeschrieben, da bereits heute die Sicherheit des Fahrzeugs ein Hauptfaktor bei der Kaufentscheidung von Neuwagen ist (Vrkljan/Anaby 2011). Zum anderen wird die Latte auch nicht sonderlich hochgelegt, bedenkt man, wie viel gefährlicher Pkw zum Beispiel gegenüber Bussen des öffentlichen Verkehrs in der Europäischen Union sind (ERSO 2019: 26).

Auch wissenschaftliche Studien sind bisweilen uneingeschränkt optimistisch. Dies gilt vor allem für jene älteren Datums. Vollautomatisierte Fahrzeuge werden als das „crashless car“ (KPMG 2012, Allesandrini et al. 2015) idealisiert und popularisiert. Sie wurden zur technologischen Entsprechung der „vision zero“, dem Ziel, tödliche Unfälle im Straßenverkehr auf null zu reduzieren. Diese Vorstellung wurde bald wegen der unveränderten physikalischen Grenzen zurückgewiesen (Winkle 2015). Selbst in Simulationsstudien, die ein gesteigertes Verkehrsaufkommen durch vollautomatisierte Car-Sharing-Fahrzeuge nachgewiesen haben, wurde dennoch darauf bestanden: „improvements in road safety are almost certain“ (ITF 2015: 6). Auch in diesem Fall wurde ein grundlegender Widerspruch übergangen. Der als „exposure“ bezeichnete Zusammenhang zwischen der Häufigkeit, mit der VerkehrsteilnehmerInnen unterwegs sind bzw. einander begegnen, und dem Unfallrisiko ist über Jahrzehnte gut dokumentiert. Mehr Aktivität führt zu mehr Unfällen (Elvik et al. 2009: 35). Schließlich wurde zu mehr Zurückhaltung aufgerufen, da durch die Vorstellung, „autonome“ Fahrzeuge würden zu absoluter Verkehrssicherheit führen, bereits Erwartungen bei zukünftigen NutzerInnen geschürt wurden, die als unhaltbar und hochproblematisch bewertet werden (Georgieva/Kolodege 2018).

Nun wird meist zurückhaltender formuliert: In Politik- und Strategiepapieren sowie Publikationen aus der technischen Entwicklung wird der Zusammenhang von Automatisierung und gesteigerter (nicht absoluter) Verkehrssicherheit nicht mehr als gegeben angenommen, sondern umgekehrt als Voraussetzung für deren Zulassung gesehen. In vielen Politikpapieren wird betont, dass die erwarteten Vorteile der Automatisierung nur über zusätzliche Vernetzung realisiert werden können (im Kontext von C-ITS - Cooperative Intelligent Transport Services; vgl. „Declaration of Amsterdam“ 2016, European Commission 2017a, STRIA 2019, Meyer 2019). Es wird auch darauf hingewiesen, dass zunächst von Potentialen gesprochen werden sollte, denen auch neue Risiken und Unsicherheiten gegenüberstehen (Feigenbaum et al. 2018). Bezüglich der Unsicherheiten wird meist der Bereich der „cyber security“ betont. Trotz der Relativierungen jüngerer Zeit bleiben die Hoffnungen groß und durch jedes technologische Add-on wird der notwendige Investitions- und Entwicklungsbedarf erhöht. So wird weiter die Einschätzung vertreten, dass der Markteintritt nicht zu weit in die Zukunft verschoben werden darf. Da Automatisierung und Vernetzung potentiell bereits Leben retten könnten (wären derart ausgerüstete Fahrzeuge bereits etwas sicherer als herkömmliche Pkw), müssen auch Kompromisse in Kauf genommen werden: „We can't wait for the perfect“ (Foxx in Shladover et al. 2019: 4). Diese Auffassung gilt für Assistenzsysteme, die FahrerInnen unterstützen, ist allerdings, wie nachstehend gezeigt wird, nicht auf jene Automatisierungsgrade zu übertragen, in denen Menschen nur mehr PassagierInnen sind.

\subsection{PERSPEKTIVE VERKEHRSSICHERHEIT: WER PROFITIERT?}

Im wissenschaftlichen Diskurs wurde mittlerweile damit begonnen, nicht alleine auf mögliche Potentiale zu fokussieren, sondern auch gezielt die technologischen Einschränkungen in den Blick zu nehmen (Mitteregger et al. 2020b, Soteropoulos et al. 2020). Ein automatisiertes Fahrsystem, das allen Fahraufgaben, die auch von Menschen gemeistert werden, gewachsen ist, wird nun auch von der Industrie, wenn überhaupt, viele Jahre in der Zukunft gesehen (Krafcik in Marx 2018). Daraus folgt, dass auch mögliche Beiträge zur Verkehrssicherheit ungleich verteilt sind. 
Die Durchsetzung von neuen Technologien und der damit einhergehende soziotechnische Wandel ist ein komplexer gesellschaftlicher Prozess (Schumpeter 1939, Geels/Schot 2007) und im Besonderen ein kommunikativer (Rogers 2003), der in der Vergangenheit immer von neuen räumlichen und sozialen Ungleichheiten begleitet wurde (Grübler 1992 sowie Beitrag 19 von Dangschat in diesem Band). Im Fall von automatisierten Fahrsystemen zeigen sich zusätzlich kleinräumliche Disparitäten, die durch unterschiedliche technologische Anforderungen von komplexen und weniger komplexen Straßenzügen bzw. Situationen entstehen. Je homogener, kontrollierter ein Straßenabschnitt ist und je mehr in dessen Instandhaltung investiert wird, desto besser ist er für automatisierte Fahrsysteme geeignet. Mit anderen Worten: Die Autobahn, am besten neugebaut, in hochentwickelten Industriegesellschaften und mit gutem Datennetz ist ihr ideales Einsatzgebiet. Langsam fahrende Shuttles, die in Erweiterung des öffentlichen Verkehrs eingesetzt werden, stellen diesbezüglich eine Ausnahme dar, setzen aber auch infrastrukturelle Begleitmaßnahmen voraus (vgl. Beitrag 14 von Allmeier et al. in diesem Band). Diese Einschränkungen können mit Verkehrsunfallstatistiken verglichen werden, um die Wirkungen des "crashless cars" zu differenzieren. Es wurde schon darauf hingewiesen, dass die Messlatte der Verkehrssicherheit für einen Einsatz als Erweiterung des öffentlichen Verkehrs ungleich höher liegt als in Fortführung der Automobilität. Das europäische Bahnsystem würde sogar in die Kategorie "ultra-sichere Systeme“ fallen, für die grundlegend andere, teils paradoxe Voraussetzungen für den Einsatz neuer Technologien gelten würden (Amalberti 2001).

Von den über 1,2 Millionen tödlichen Straßenverkehrsunfällen, die immer wieder als Referenz für den gesellschaftlichen Nutzen von automatisierten und auch vernetzten Fahrsystemen bemüht werden, sind junge, arme Menschen in Länder mit niedrigem und mittlerem Einkommen überproportional betroffen. Auf diese Gruppe entfallen 90 Prozent der Verkehrstoten weltweit (WHO 2015: 4). Am schlimmsten ist die Situation im afrikanischen Raum, vor allem in Anbetracht des relativ geringen Motorisierungsgrades. Mit einem Anteil von insgesamt über 43 Prozent an den im Straßenverkehr tödlich verunglückten Personen sind Fußgängerlnnen und RadfahrerInnen die gefährdetste Gruppe (WHO 2015: 8).

Abbildung 1: Symbolbild der WHO für besonders gefährdete Personen im Straßenverkehr und Googles Testbetrieb in Chandler, Arizona
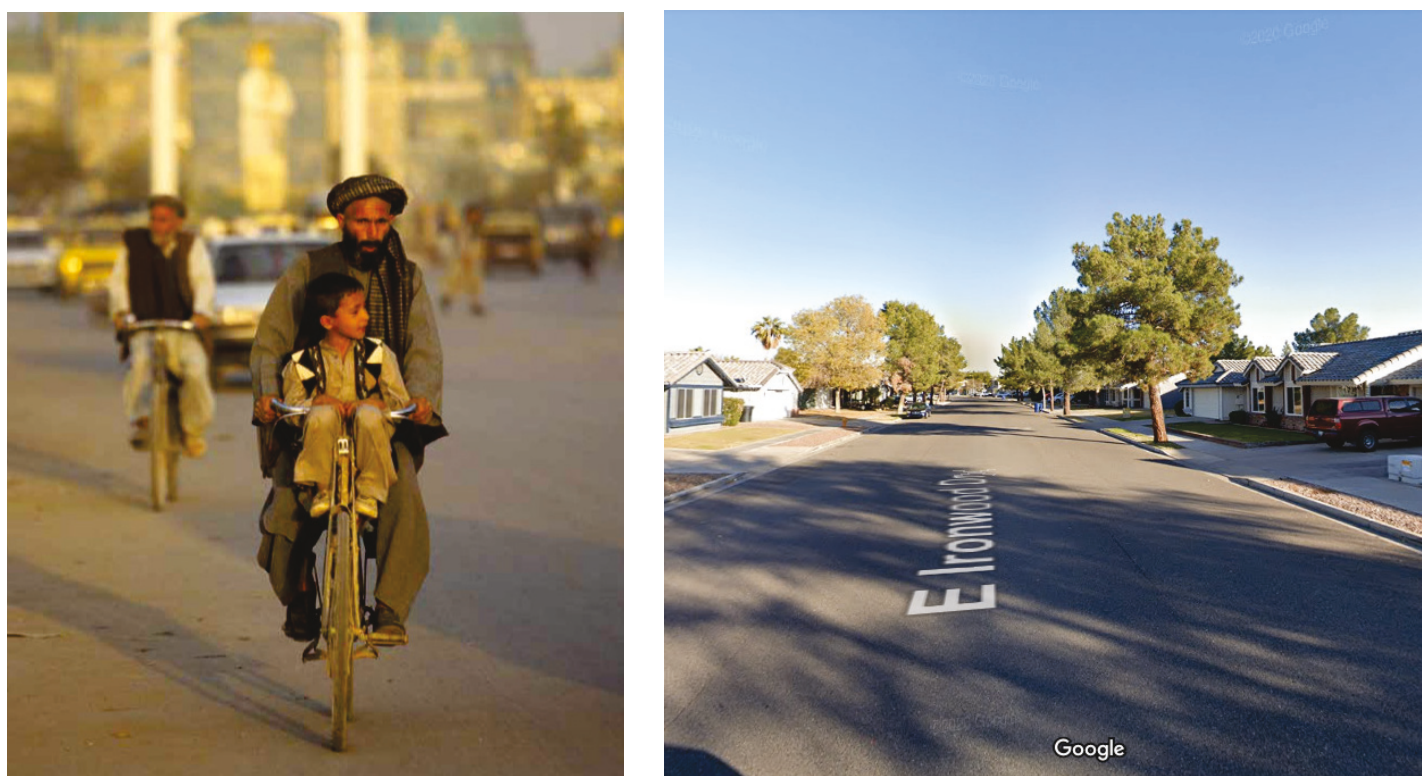

Die hier abgedruckten Bilder sind explizit von der Creative Commons Lizenz des Textes ausgenommen. Die Rechte bleiben bei den Verfassern. Quelle links: WHO (2015: VIII); Foto rechts: (Google 2020) 


\section{Automatisierung}

Die Entwicklung von automatisierten und vernetzten Fahrzeugen läuft diametral gegen diese Ausgangslage. In den am stärksten betroffenen Ländern und Regionen werden weder bedeutende Tests durchgeführt noch gibt es virtuelle Testumgebungen, mit denen lernende Algorithmen trainiert werden könnten. Um Sensorik zu entwickeln, existiert ein einziger öffentlich zugänglicher Datensatz, der wiederum ausschließlich auf optische Sensoren ausgelegt ist (Mitteregger et al. 2020a, Kang et al. 2019).

Auch was mögliche Einsatzgebiete in bestehenden Straßennetzen betrifft, ist eine Differenzierung möglich. In Österreich, einem Spitzenreiter hoher Verkehrssicherheit, entfallen auf Autobahnen und Schnellstraßen 8,8 Prozent der Verkehrstoten. Der Großteil tödlicher Unfälle ereignet sich auf ehemaligen Bundesstraßen (204 Tote), Landesstraßen (104) und sonstigen Straßen (66; BMI 2020). Die Gesamtlänge des Straßennetzes in Österreich 2010 betrug 114.590 Kilometer, davon waren etwa rund 2.185 Kilometer Autobahnen oder Schnellstraßen, was einem Anteil von ca. 2 Prozent entspricht (BMVIT 2012). Außerdem zeigt sich in Österreich und vor allem auch anderen Ländern mit hoher Verkehrssicherheit, dass die Sicherheit für Verkehrsteilnehmerlnnen innerhalb von Fahrzeugen zu-, aber für Verkehrsteilnehmerlnnen außerhalb eines motorisierten Verkehrsmittels abgenommen hat oder gleichgeblieben ist. So sind zum Beispiel die tödlichen Unfälle von FahrradfahrerInnen auch in Österreich in den letzten Jahren steigend, während sie für Pkw rückläufig sind (Statistik Austria 2017: 11). Die Unfallrate, die Zahl der Unfälle je zurückgelegter Distanz, liegt für Fußgängerlnnen 6,7-mal und für FahrradfahrerInnen 9,4-mal höher als für Lenkerlnnen eines Pkw (Elvik 2009: 56).

Zusammenfassend kann gesagt werden, dass mit hohem technologischem und ökonomischem Einsatz mittelfristig eine Steigerung der Verkehrssicherheit nur in ohnedies privilegierten Ländern und hier wiederum in einem Bruchteil des Straßenverkehrsnetzes wahrscheinlich ist. Echte globale Probleme der Verkehrssicherheit liegen nach heutigem Maßstab völlig außerhalb der anzunehmenden Entwicklung automatisierter Fahrsysteme. Auch sind keine ernsthaften Anstrengungen sichtbar, die tatsächliche Ausgangslage in der Technologieentwicklung zu berücksichtigen.

\section{Vernetzung}

Die Vernetzung von Fahrzeugen soll in zwei Bereichen die Verkehrssicherheit steigern. Erstens sollen automatisierte und vernetzte Fahrsysteme bei der Umfelderfassung unterstützt werden. In bestimmten Streckenabschnitten (z. B. Kreuzungen, Autobahnfahrten, Baustellenbereichen) oder unter Bedingungen, auf die ein Fahrsystem nicht ausgelegt ist (z. B. Schnee, Regen oder während Unfällen), ergänzen die in der Infrastruktur verbauten Sensoren oder jene anderer Fahrzeuge Umgebungsinformationen, damit die Fahraufgabe weiter bewältigt werden kann (Carreras et al. 2018, STRIA 2019). Der Einsatz der Sensorik wird aktuell vor allem auf Autobahnen diskutiert. Das Ziel ist, die Eignung des Streckennetzes gezielt zu heben bzw. Lücken auf einzelnen Routen durch infrastrukturelle Investitionen zu schließen (Abb. 2). Verfällt zum Beispiel die Tauglichkeit für automatisierte Fahrsysteme in einem Abschnitt einer prinzipiell für den Einsatz von automatisierten Fahrsystemen geeigneten Strecke, springt die Infrastruktur ein, um zu kompensieren (Abb. 2 rechts). Die Vernetzung des Fahrzeugs und geeignete Sensoren als Teil der digitalen Infrastruktur sind hierfür Voraussetzung.

Obwohl noch ein einheitlicher Standard noch fehlt, werden Streckenabschnitte schon teilweise mit Sensorik ausgestattet, um sogenannte „Day-1-Services“ (Informationen u. a. über Baustellen oder defekte Fahrzeuge am Straßenrand) zu realisieren. Dabei werden Informationen über das vernetzte Fahrzeug an den/die Fahrerln weitergegeben (ASFINAG 2019, European Commission 2017a).

Day-1-Services bereiten auch den zweiten Bereich vor, in dem Vernetzung die Verkehrssicherheit automatisierter Fahrzeuge unterstützen soll. Sie ermöglicht die umfassende Dokumenta- 
Abbildung 2: Ertüchtigung eines Streckenabschnittes für automatisierte Fahrsysteme durch digitale Infrastruktur

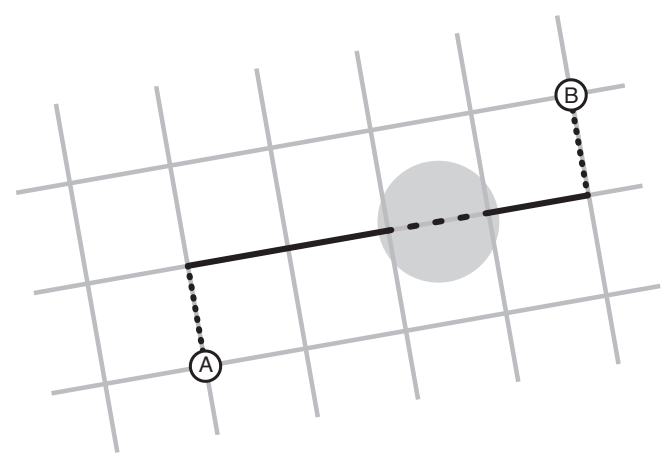

Ein Teilstück der Strecke ist nicht automatisierungstauglich.

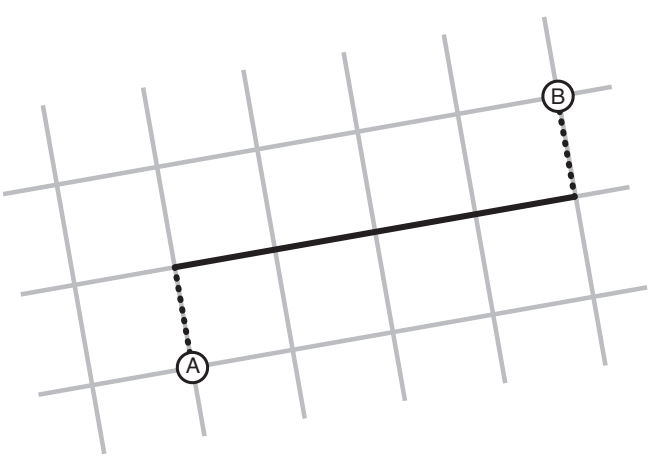

Nach einer Infrastrukturanpassung ist die Strecke durchgehend automatisiert befahrbar.

Quelle: eigene Darstellung nach Alkim in STRIA (2019: 21)

tion von Unfällen oder Vorfällen (und die Reaktion des Fahrsystems darauf) ${ }^{2}$ bzw. in fernerer Zukunft live den aktuellen Zustand des Umfelds und des Fahrsystems. Durch die Kommunikation der Fahrsysteme untereinander oder über eine Leit- bzw. Kontrollstelle könnten aktuelle Informationen ausgetauscht und in der Verkehrssteuerung bzw. der Routenplanung berücksichtigt werden. Zudem lernen automatisierte und vernetzte Fahrsysteme voneinander (Casademont et al. 2019). So wäre es möglich, dass automatisierte und vernetzte Fahrzeuge eines Herstellers, eines Verkehrsträgers oder das Verkehrssystem als Ganzes Schritt für Schritt optimiert werden würden. Derart vernetzte Systeme wären Menschen mit jedem gefahrenen Kilometer immer weiter überlegen, da - so die Vision - „[a]ll the unborn cars get born with the full wisdom of their forefathers" (Thrun in Shakland 2016).

Die dafür notwendige digitale Infrastruktur würde jedenfalls erhebliche Kosten verursachen, die über die öffentliche Hand an die Allgemeinheit weitergegeben werden könnten (Polis 2018, Mitteregger et al. 2019). Eine vergleichbare Dynamik der Externalisierung der Kosten eines elitären Systems stand auch am Beginn des Automobilzeitalters (McShane 1994: 203-228). Auch aufseiten der digitalen Infrastruktur wurden bislang vor allem die Probleme im Bereich der Cybersecurity hervorgehoben (Landini 2020).

\subsection{EIN GANZ NEUER WEG}

"The future of this new technology is so full of promise. It's a future where vehicles increasingly help drivers avoid crashes. It's a future where the time spent commuting is dramatically reduced, and where millions more including the elderly and people with disabilities-gain access to the freedom of the open road. And, especially important, it's a future where highway fatalities and injuries are significantly reduced" (Elaine L. Chao in NHTSA 2017: i).

2 Ob die Reaktionsmechanismen und Entscheidungsgrundlagen eines so komplexen und auf lernende Algorithmen basierenden Systems wie eines hochautomatisierten Fahrzeuges oder einer vernetzten Flotte jemals eingesehen werden können, scheint fraglich (Castelvecchi 2016). 
Der Weg, der mit Automatisierung und Vernetzung von Straßenfahrzeugen begonnen wurde, wird als ein grundlegend neues Konzept der Verkehrssicherheit beschrieben. Das neue Ziel ist, durch technische Systeme Unfälle zu vermeiden, bevor diese eintreten (vgl. Rosekind in Shladover et al. 2019: 4). Im Kern betrifft dies den Übergang von passiven zu aktiven Sicherheitssystemen: Passive Sicherheitssysteme wie Gurt, Stoßstange oder Airbag mindern die Folgen eines Unfalls (für die InsassInnen), aktive Sicherheitssysteme wie Notbremsassistent oder Adaptive Cruise Control verhindern den Unfall.

Abbildung 3: Sicherheit als Panzer (passive Sicherheit) und Sicherheit als Vorsicht (aktive Sicherheit)

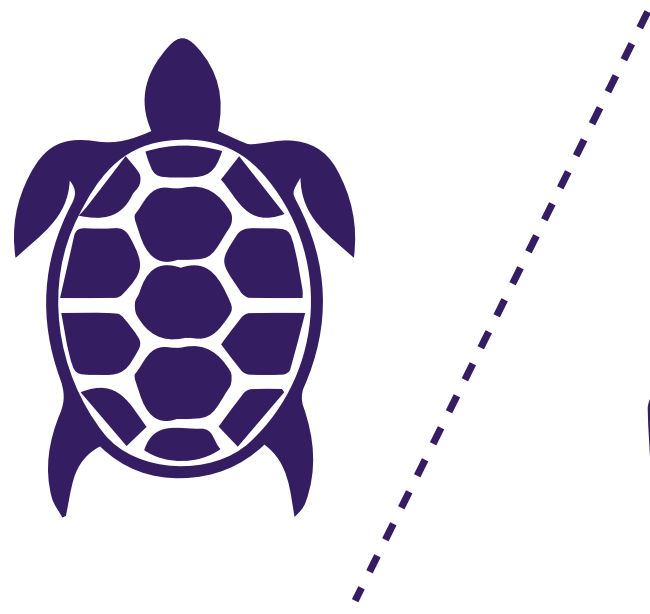

war

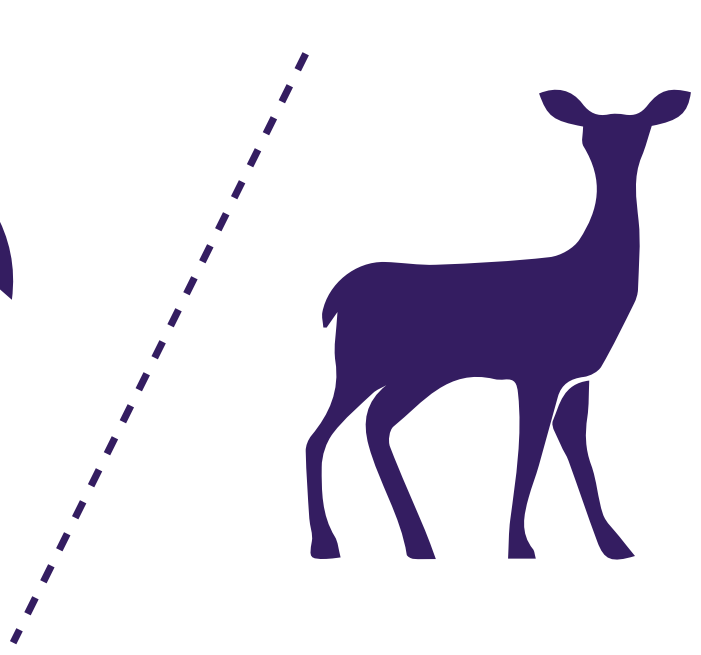

wird

Quelle: eigene Darstellung

Diese Logik entspricht jener der Luftfahrt, bei der dieses Prinzip umfassend eingesetzt wird. Für die Sicherheit der PassagierInnen im Falle potentiell katastrophaler Ereignisse spielen Gurt oder Beschaffenheit des Rumpfes eine untergeordnete Rolle. Die hohe Sicherheit von zivilen Linienflügen wird vor allem erreicht, weil ein System geschaffen wurde, das die wesentlichen externen Risikofaktoren erfassen kann und bekannte Unfallursachen durch menschliches Fehlverhalten verhindert. Die Hauptbestandteile dieses Systems sind eine lückenlose Flugverkehrskontrolle und umfassende Wetterdaten. Konkret bedeutet dies, dass die PassagierInnen nicht in erster Linie für das Durchfliegen einer Gewitterzelle entsprechend gesichert werden, sondern, dass eine Gewitterzelle erkannt oder antizipiert und umflogen wird. Zudem unterstützen umfassende Assistenzsysteme die Pilotlnnen, denen in vielen Fällen nur mehr eine überwachende Rolle zukommt. Was würde diese Logik für den Straßenverkehr und im Besonderen für den Straßenraum bedeuten?

Automatisierte und vernetzte Fahrsysteme befinden sich bislang erst in der frühen, formativen Phase der Technologieentwicklung (Anderson/Tushman 1990; Bergek et al. 2008; Mitteregger et al. 2020b: 69). Sprechen wir heute über die Wirkungen von automatisierten und vernetzten Fahrzeugen, dann wird eine Diskussion um die Potentiale von konzeptionellen Designs geführt: Deren tatsächliche Funktion entspricht dem, was man für diese frühe Entwicklungsphase erwarten würde. Sensoren sind selbst unter Idealbedingungen in den meisten Aspekten den menschlichen Fähigkeiten entweder gleichwertig oder unterlegen. Dazu nimmt ihre Funktionsfähigkeit stark ab, wenn sich die Umfeldbedingungen vom idealen Zustand (gute Sicht, deutlich erkennbare Bodenmarkierungen etc.) entfernen (Schoettle 2017). Aber auch unter guten Bedingungen ist die Performance noch eingeschränkt: Eine Studie, die Tests in Kalifornien untersucht hat, konnte nachweisen, dass Auffahrunfälle bei Tests wahrscheinlicher sind, wenn das 
automatisierte Fahrsystem aktiviert ist und nicht menschliche SicherheitsfahrerInnen am Steuer sind (Boggs et al. 2020). Diese medial und öffentlich ausgetragene Diskussion ist deswegen relevant, weil bereits in dieser frühen Phase in der Diffusion neuer Technologien grundlegende Funktionsweisen ausgehandelt werden (Rogers 2003, Foucault 1981, vgl. Beitrag 4 von Manderscheid sowie Beitrag 19 von Dangschat in diesem Band).

Die Verkehrssicherheit betreffend zeigen sich im aktuellen Diskurs zusammenfassend folgende grundlegende Ansprüche an automatisierte und vernetzte Fahrsysteme.

1. Den Konzepten automatisierter und vernetzter Fahrsysteme werden hohe theoretische Fähigkeiten (Potentiale) im Bezug zur Verkehrssicherheit unterstellt.

2. Diese Unterstellung wird von den beiden Annahmen getragen, dass automatisierte Fahrsysteme:
a. zuverlässiger als menschliche Fahrerlnnen funktionieren könnten und
b. den Menschen kognitiv überlegen sein werden.

3. Vernetzung könnte die Umfelderfassung erweitern und mögliche Schwächen eines automatisierten Fahrsystems kompensieren.

4. Schließlich wird durch Vernetzung umfassendes Reporting (von Unfällen, Fast-Unfällen, Vorfällen und Fast-Vorfällen) möglich, wodurch alle Teile eines Systems voneinander lernen und die Performance weiter gesteigert würde.

Die Vorstellung eines automatisierten und vernetzten Verkehrssystems als perfektes passives Sicherheitssystem würde einen tatsächlichen Paradigmenwechsel bedingen. Dieser wurde von der Europäischen Kommission klar formuliert: Verkehrssicherheit (und Verkehrsfluss) wurden für lange Zeit von FahrerInnen und anderen VerkehrsteilnehmerInnen organisiert. Das System beruhte darauf, dass Verkehrsregeln eingehalten oder verkehrssteuernde Maßnahmen befolgt werden. Der automatisierte und vernetzte Verkehr stellt diese Logik auf den Kopf: Ein Bottomup-System wird zu einem Top-down-System (STRIA 2019: 8)³.

Es ist undenkbar, dass eine derart grundlegende Neuorientierung nicht zu einem ebensolchen Wandel des Straßenraums führen würde. Dieses auf Perfektion ausgelegte Sicherheitssystem würde Einfluss auf alle anderen Tätigkeiten haben, die im Straßenraum über den Transport hinaus sonst so gemacht werden. Das ist der Unterschied zur Luftfahrt, deren Sicherheitssysteme übertragen werden. Dieser Aspekt des automatisierten und vernetzten Straßenverkehrs betrifft nicht allein Verkehrssicherheit im engeren Sinn. Zur Debatte steht das Prinzip, das eine Gesellschaft wählt und mit dem Sicherheit im öffentlichen Raum der Straße erreicht werden kann. Davon sind vielfältige Teilbereiche betroffen, die nicht allein auf eine verkehrliche Betrachtung reduziert werden können und auch nicht - wie das häufig problematisierte Feld der Cybersecurity - technologisch lösbar sein werden.

3 Im Wortlaut: „In road transport, e.g. where safety and efficiency have been organized for long time with the driver and other road users in charge of complying with traffic rules and traffic management, connected and automated road transport turns this concept from bottom-up to top-down: If the electronic control systems embedded in the vehicle take decisions instead of the human driver, the cognitive capabilities of an automated vehicle are determined by the performance of its perception systems, algorithms and knowledge base“ (STRIA 2019: 8). 


\section{STRASSEN: TRANSPORT UND LEBENSRAUM}

Den Straßenraum alleine auf die Funktion der physischen Zirkulation zu beschränken ist ein Reduktionismus mit bekanntermaßen weitreichenden Folgen. Straßen sind auch wertvoller Lebensraum und durch diese doppelte Bedeutung untrennbar an das Konzept von Stadt selbst gebunden (Marschall 2009). Die Bedeutung, die Straßen in der Betrachtung der Geschichte der Stadt zugeschrieben wurde, wird nachstehend umrissen.

Die Bewegung von Dingen durch und in den Straßen ermöglicht den Metabolismus von dichten menschlichen Siedlungen: Er hat eine vor allem biologische Komponente, wenn es um den stetigen Fluss von Konsumgütern geht. Er hat aber auch eine stärker kulturelle Komponente, wenn es um den Austausch von Werken geht, die darauf ausgelegt sind, ihre HerstellerInnen zu überleben. Und schließlich leben Städte nicht nur von der Bewegung von Dingen. Der Fluss von Ideen in und durch Straßen ermöglicht das kommunikative Hinterfragen und das Schaffen eigener Verhältnisse: die gesellschaftliche Komponente, die im Austausch mit dem Anderen, dem Reisenden entstehen (Arendt 1958; Reki 2004; Simmel 1908: 509-512). Der über Straßen organisierte Fluss von Menschen, Dingen und Ideen (und das Stocken dessen) erhält Städte und Stadtgesellschaften und macht es notwendig, die eigene Position ständig neu zu bestimmen.

Ohne den öffentlichen Raum der Straße wären dichte Siedlungen unbewohnbar oder sie müssten, wie von ArchäologInnen freigelegte alternative Konzepte zeigen, grundlegend neu gedacht werden (Hodder/Pels 2010). Auch hier gibt es einen funktionalen Aspekt, weil Bewohnerlnnen in dichten urbanen Räumen attraktive öffentliche Räume brauchen (deren Bedeutung angesichts der globalen Klimakrise weiter zunimmt und während der Covid19-Pandemie wieder erkannt wurde), die als Erweiterung des Wohn- und Lebensraums zum Sitzen, Reden oder zum Spielen genutzt werden können (Gehl 2009, EEA 2009). Diese beiden häufig in Konkurrenz stehenden Anforderungen an den Straßenraum - Transport auf der einen und Aufenthalt auf der anderen Seite - prägen seit jeher die Entwicklung von Straßen und Städten. Dieser Nutzungskonflikt führt aber letzten Endes dazu, dass Straßen als ,institutionalisiere menschliche Bewegung" gesehen werden können (Rykwert 1986) und ihre Gestalt und Nutzung dominante Machtstrukturen, Identitäten und Lebensweisen offenbaren (Sheller/Urry 2006, Cresswell 2011: 551).

\subsection{DIE BEDEUTUNG DES STRASSENRAUMS FÜR URBANITÄT}

"[C]ities are their streets. Streets are not a city's veins but its neurology, its accumulated intelligence" (Gopnik 2016).

Die Bedeutung von Straßen als Lebensraum ist über die funktionale Zuschreibung im Sinne der Lebensqualität nicht ausreichend erfasst. Seit der Antike werden Straßen als Teil des öffentlichen Raums gesehen, wodurch die Stadt („polis“) erst von einer Ansammlung an Steinen („urbs“) zu einer Gemeinschaft von Menschen („civitas“) wird, die nach bestimmten Prinzipien handelt (Fustel de Coulanges 1979). Den öffentlichen Raum der Straße zu betreten, bedeutet, die kontrollierte Sicherheit des privaten Raums zu verlassen, denn was öffentlich ist, kann betrachtet, kritisiert und modifiziert werden - vorausgesetzt, es wird von der Öffentlichkeit wahrgenommen (Arendt 1958: 95). So wird der soziale Raum der Straße ständig neu erzeugt und verändert (Massey 2005). Er wandelt sich mit den Akteurlnnen im Tagesverlauf, während der 
Jahreszeiten, durch Gesetze und eben auch mit Technologien, die neue Arten der Lebensführung ermöglichen (Gerhardt 2012: 32f.). Die „offene, leicht wandelbare Natur von Straßen“ (Appleyard 1987: 1) liegt hierin begründet.

Als Teil des öffentlichen Raums ist die Straße der Ort für das formalisierte und spontane Geschehen von sich wandelnden Stadtgesellschaften - wo Hinrichtungen, Musik, Protest, ein Fußballspiel oder Liebe stattfinden können. Jede Neugestaltung der Straße hat demnach immer Folgen für die Stadt als Ganzes und deren Gesellschaft. So wird die Straße zum Schauplatz kultureller Kämpfe: Wo das Recht zu protestieren eingeschränkt, der Zugang zum Straßenraum für Teile der Gesellschaft verstellt oder der Raum zwischen Fußgängerlnnen, Radfahrerlnnen und Autos neu verteilt wird, ist das Konzept der Stadt als Ganzes betroffen. Dementsprechend ist ein kritischer Diskurs gegenüber neuen Technologien im öffentlichen Raum mehr als geboten, da sie, mit lokalen Eigenheiten, den Straßenraum durch Platzansprüche, Emissionen und notwendige neue Regelwerke global verändern können. Das Auto ist hierfür das bekannteste Beispiel.

\subsection{SICHERHEIT UND ÖFFENTLICHKEIT}

Auf der Suche nach "anthropologischen Gemeinsamkeiten des Mobilitätsverhaltens“ bedient sich Cesare Marchetti eines biologischen Determinismus: Menschen leben mit einer inhärenten Spannung, die zwischen einem „Höhlentrieb“ auf der einen und dem „grundlegenden Trieb [...,] ihr Territorium zu erweitern“, auf der anderen Seite entsteht (Marchetti 1994: 75). Aus diesem Grund sei jedes Verlassen der Höhle „aufwendig“, da das Streben nach außen nicht nur mit körperlicher Anstrengung verbunden ist, sondern auch die "Gefahr [,]von Raubtieren oder Feinden attackiert zu werden“, lauert (ebd.). Diese düstere Sicht auf das menschliche Dasein wirft die Frage auf, wie mit derartigen Trieben ausgestattete Tiere Siedlungen schaffen und von Dörfern zu Metropolen weiterentwickeln konnten, die Marchetti im Verlauf seines Textes dann auch zur Beweisführung verwendet.

Einige der erschreckendsten Szenen der Literatur basieren darauf, dass die „wandelbare Natur der Straße" umschlägt, die Masse zur Meute wird und sich gegen den/die Einzelne/n oder eine Minderheit wendet. Und auch die Freiheit des öffentlichen Raums bleibt immer ein Privileg, das nie allen zuteil werden kann (Arendt 1958: 51). Die grausame Realität, die daraus entsteht, ist, dass sich obdachlose Personen, Mitglieder von Minderheiten oder diskriminierte Personengruppen zwar im öffentlichen Raum befinden, ihre Anwesenheit aber ignoriert und ihr Handeln und auch ihre Sicherheit dementsprechend eingeschränkt werden (vgl. Simmel 1903).

Marchettis Vorstellung eines Hobbes'schen Naturzustands der Mobilität bleibt fragwürdig. Die so gestützte Theorie von konstanten Zeitbudgets ist allerdings im Diskurs zu möglichen Effekten von automatisierten Fahrzeugen wieder aufgetaucht (Almeida Correia et al. 2016, Maia/Meyboom 2018, Newman et al. 2016). Was sie aber ungeachtet der ihr innewohnenden Agoraphobie zeigt, ist die Bedeutung von Sicherheit für die Nutzung des öffentlichen Raums, denn so bedeutend die Straße als Transport- und Lebensraum für Städte ist, so fragil bleibt der Schutz, den sie bietet.

„[D]ie Straße war immer der Ort des [...] Konflikts, zwischen Barriere und Zugang, zwischen Bewohner und Reisenden, zwischen dem Leben auf der Straße und dem drohenden Tod" (Appleyard 1987: 9). Entgegen Marchettis These wurde die Exponiertheit oder Sichtbarkeit, die mit der Öffentlichkeit notwendigerweise verbunden ist, mit einer bestimmten Form von Sicherheit in Verbindung gebracht - eine Form von Sicherheit, die auf Dichte und Diversität beruht. Sind nicht Menschen soziale Tiere, die alleine nicht lebensfähig sind (Aristoteles Pol. 1253a1-11)? Und würde man nicht die von Cafés und Bars belebte Straße am nächtlichen Heimweg in den allermeisten Fällen der dunklen Gasse vorziehen? 
Immanuel Kant ging soweit, dass er die Öffentlichkeit zum konstituierenden Prinzip seiner Philosophie erklärte. Demnach funktioniert die Öffentlichkeit als kritisches „Publikum“ und deckt jenes Verhalten auf, demnach der/die Einzelne zum eigenen Vorteil handelt und andere in ihrem Handeln einschränkt oder gefährdet. „Lichtscheu“, so Kant, ist alles, was nur im Privaten getan werden muss: Wenn es öffentlich werden würde, bestünde das Risiko, dass „der Widerstand Aller gegen meinen Vorsatz gereizt" wird (EwF 391, EwF 386; Gerhardt 2012: 163f.). Dazu ist es notwendig, dass man einander gleichberechtigt begegnet. Sich in die Öffentlichkeit zu begeben, bedeutet, ein gewisses Risiko einzugehen, denn ich selbst werde auf die Aufmerksamkeit der Anderen angewiesen sein und meine Handlungen werden kritisch geprüft. Im Gegenzug kontrolliere ich mit meiner Aufmerksamkeit, was geprüft und wer geschützt wird. Nur wo der überwachende Blick erwidert werden kann, herrschen gleiche Verhältnisse.

\subsection{AUGEN AUF DER STRASSE}

Die in der Stadtplanung bekannteste Vertreterin der Position, dass Sichtbarkeit, Sicherheit und Öffentlichkeit ineinandergreifen, ist Jane Jacobs. Sie erinnert ihre Leserschaft daran, dass sichere Straßen nicht das Produkt eines zentralisierten Machtsystems sein können, sondern von den Individuen geschaffen werden, die sie nutzen. „Gehsteige und diejenigen, die sie benutzen, sind keine passiven Begünstigten von Sicherheit oder hilflose Opfer von Gefahren. Bürgersteige, ihre angrenzenden Nutzungen und ihre Nutzer sind aktive Teilnehmer am Drama der Zivilisation versus Barbarei in den Städten“ (Jacobs 1961: 30). Für Jacobs bedarf es der „Augen auf der Straße" vor allem durch jene Personen, die sich im Straßenraum und auch in den anliegenden Gebäuden befinden (ebd.: 35). Gemeinsam sorgen sie für die Sicherheit, die ein Leben in der Straße - und damit in der Stadt - erst ermöglichen.

Auch Jacobs hat ein paritätisches Prinzip zugrunde legt: Die Öffentlichkeit selbst schafft das zivilisierte Miteinander. Wo das gleichberechtigte Miteinander gebrochen wurde, „kann keine noch so große Zahl an Polizisten das zivilisierte Zusammenleben wiederherstellen“ (ebd.: 31). In diesem Beharren auf dem Prinzip der Gleichheit zeigt sich Jacobs' demokratische Grundhaltung - und auch wenn sie darauf besteht, dass dies auch für die Straßen der New Yorker Upperclass gilt. Alle möglichen Bediensteten, Portiere oder Hundesitter (heute sind es vermehrt Überwachungskameras) bevölkern hier den Straßenraum; sie tun dies allerdings nur, weil sie dafür bezahlt werden. Tatsächlich, so Jacobs, fehlen diesen Orten die Gründe, die irgendjemand hier aus freien Stücken in den Straßenraum ziehen würden (ebd.: 40). Der entscheidende Punkt ist, dass Sicherheit nur zu „Zivilisation“ führen kann, wenn diese von der Öffentlichkeit erzeugt und nicht von einem institutionalisierten Machtapparat durchgesetzt wird. Alle technischen Mittel oder institutionellen Körper verletzen dieses Prinzip.

Sartre legt in Sein und das Nichts eine detaillierte Argumentation vor, die Jacobs später angestellten Beobachtungen in den Straßen des Greenwich Village in New York entspricht. Sartre beharrt darauf, dass Gleichheit und Freiheit nur dort existieren, wo Blicke erwidert werden (1962: 356). Diese Machtdynamik verlässt das Gleichgewicht, wenn eine Person zum Beispiel durch ein Schlüsselloch späht und sieht, ohne gesehen zu werden. Der architektonische Ausdruck dieses Prinzips ist das Panopticon, dass durch Foucault zur Ikone moderner Überwachungsmechanismen wurde (Foucault 2012).

\subsection{DAS ENDE GLEICHER VERHÄLTNISSE}

Jacobs' Abneigung gegen den Pkw ist eng mit dieser Argumentation verbunden. Berühmt wurde ihre Teilnahme an den Protesten gegen Stadtautobahnen wie den Lower Manhattan Expressway, den der damalige Stadtplaner Robert Moses durch Manhattan schlagen wollte (Gratz 2010). 
Jacobs' Beharren auf den Zusammenhang von gleichberechtigter Sichtbarkeit, Öffentlichkeit und Sicherheit macht deutlich, dass eine auf das Auto ausgerichtete Stadtstruktur nicht nur die Verkehrssicherheit im engeren Sinn betrifft, sondern es zeigt sich auch ein tiefer greifender Effekt, der für "die vielleicht größte Transformation der Stadt in den letzten tausend Jahren“ (Marshall 2005: 3) verantwortlich war: Der motorisierte Individualverkehr hat dazu geführt, dass das paritätische Prinzip des öffentlichen Raums in so gut wie allen Städten untergraben wurde. In einem Auto nimmt man die Menschen im Straßenraum natürlich wahr, aber jede visuelle Begegnung wird drastisch verkürzt. Die Gestalt des Menschen im Fahrzeug ist durch die Reflexion der Scheiben für andere teilweise verborgen. Stimmen von außen können durch das Chassis des Fahrzeugs höchstens gedämpft wahrgenommen werden, alles an Geräuschen, die ins Innere gelangen, muss gegenüber dem Geräusch des Motors bestehen. Der Körper der Person im Fahrzeug ist geschützt, beansprucht mindestens zehn Quadratmeter, während alle anderen Tonnen von Stahl ausgesetzt sind. Unter diesen ungleichen Voraussetzungen verschwand die bis zum motorisierten Individualverkehr bestehende Beziehung, in der die belebtesten Straßen immer auch die wichtigsten sozialen Orte der Stadt waren (ebd.: 3f.).

Abbildung 4: Lower Manhattan Expressway, New York City (Modell)

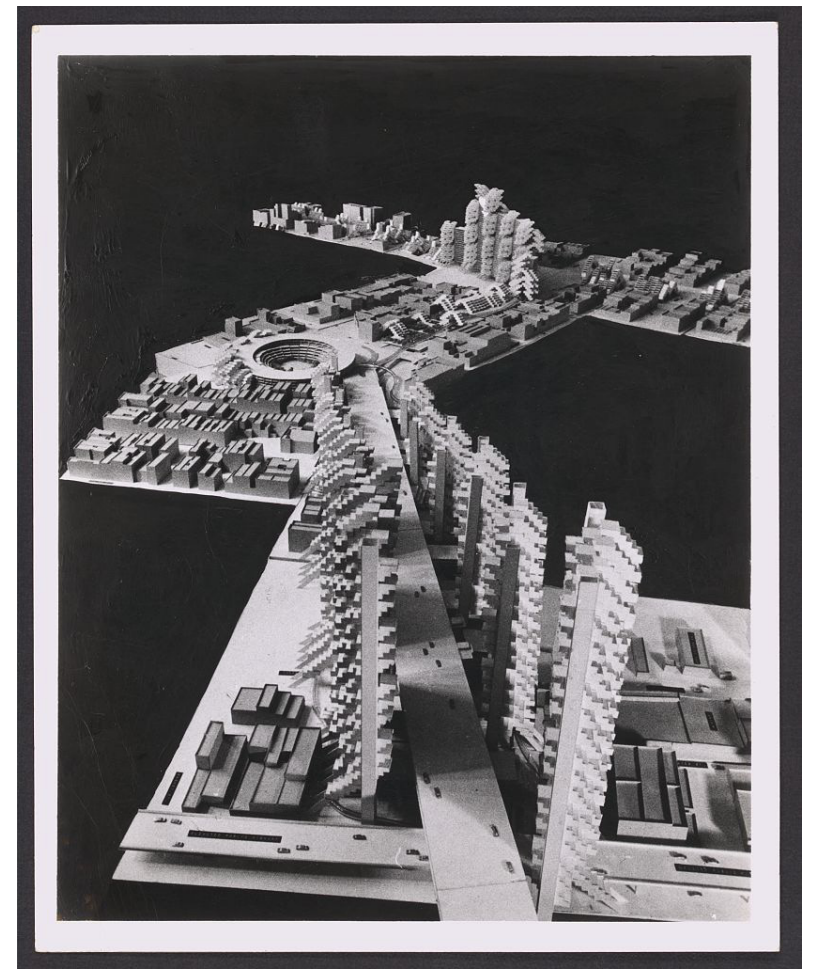

Foto: Paul Rudolph, Lower Manhattan Expressway, New York City, ca. 1970. Modell, Perspektive; Library of Congress, www.loc.gov/item/2010647138/.

Ein ernsthafter Versuch, die durch das Auto verlorene Sicherheit der Straße wiederherzustellen, beträfe dementsprechend nicht allein die Reduktion von Verkehrsunfällen allein: Es ginge um die Wiederherstellung gleichwertiger Verhältnisse in großen Teilen des Straßennetzes und damit einer räumlichen Situation, in der Sicherheit das Entstehen von Öffentlichkeit stützt. Der Blick auf die Vergangenheit weist allerdings in eine andere Richtung. Da wechselnde Begriffe der Freiheit von wechselnden Mobilitätsmöglichkeiten begleitet wurden, gingen durch die Geschichte neue Mobilität und neue Formen der Überwachung Hand in Hand. 


\title{
3. VON ÜBERWACHUNG ZU „SOCIAL ENGINEERING“
}

\begin{abstract}
"[The] movement of persons and of ,things' (goods) will become the focal points of the transport system. All people will be connected to the transport system, as will be all goods (via the ,Internet of Things'), and they will collect and share information" (European Commission 2017b: 10).
\end{abstract}

Im Feudalismus wurden all jene, die keinem Herren zuzuordnen waren, mit Brandzeichen versehen. Statistische Methoden der Volkszählung und Gefängnisse verbreiteten sich zu einer Zeit, als Nationalstaaten entstanden sind und die Mobilität auch für Arme zunahm. Dem Pkw wird nachgesagt, dass er als privater Raum auf öffentlichem Gut dazu beigetragen hat, dass die Justiz sich immer weiter in die vormals privaten Lebensbereiche vorgewagt hat (Cresswell 2006, Foucault 2007, Seo 2019).

Mit steigender Mobilität wurde so Schritt für Schritt das auf Gegenseitigkeit basierende Prinzip der sozialen Kontrollen in Gesellschaften von Institutionen und technischen Innovationen übernommen. In einem Zukunftsszenario, das als Teil in Mobilities veröffentlich wurde (Urry 2007), denkt John Urry über automatisierte und vernetzte Fahrzeuge und die Mobilität in einem „digitalen Panoptikon" nach. Später hatte Urry dieses Szenario angesichts der globalen Klimakrise auch für „zunehmend notwendig“ erachtet (Adey/Bissel 2010: 6) und darüber spekuliert, dass Singapur der erste Ort sein könnte, an dem dieser Zustand erreicht werden könnte. Entgegen Urrys These wird hier argumentiert, dass der Treiber für ein solches Szenario nicht die plötzliche Wertschätzung des fragilen Ökosystems sein könnte, sondern eine Reformation der Verkehrssicherheit.

\subsection{DAS NIE PERFEKTE SYSTEM}

Wie würde so ein System aussehen? Wie auch Urry betont, wäre dieses automatisierte und vernetzte Verkehrssystem exklusiv (vgl. Urry 2008: 273f.). Wie oben angeführt, wären erstens die erheblichen Investitionen aufseiten der öffentlichen Hand ein begrenzender Faktor und zweitens limitiert die mittelfristige technologische Machbarkeit den Einsatz auf einen Bruchteil des existierenden Straßennetzes. Für John Urry wird mit der Automobilität zugunsten des Klimas gebrochen. Die hier rekonstruierte Entwicklungslinie legt keine derartige Transformation nahe.

Automatisierung und Vernetzung tragen dazu bei - so der aktuelle Diskurs -, dass etablierte Grenzen - wie zwischen dem öffentlichen und dem Individualverkehr und auch zwischen Güter- und Personenverkehr - im Zuge einer „Hybridisierung“ verschwinden (Lenz/Fraedrich 2015, Mittereggger et al. 2020b: 44). Letzten Endes führt dies dazu, dass der heute grundlegenden Frage „Welches Verkehrsmittel wählen Personen für einen bestimmten Weg?“ die Frage „Erfordert ein Weg die Anwesenheit eines Menschen oder wird dieser von Maschinen delegiert?" vorgelagert wird (Mitteregger et al. 2020b: VIII). Bereits heute (und verstärkt durch die Covid19-Pandemie) sind vielfältige mobile Roboter im Einsatz, mit denen Menschen nicht nur Wege, sondern auch Aufgaben - und hier vor allem im Bereich der Sicherheit - an Maschinen delegieren (Mitteregger 2020). Dabei entstehen die oben beschriebenen technologischen Einschränkungen durch die Komplexität des Straßenraums, gelten für diese kleinen, langsam fahrenden, auf Gehwegen eingesetzten Roboter aber nicht oder nur in viel geringerem Maße.

Dies ist allerdings kein „Trendbuch“, sondern der Exzess bestehender Prinzipien. Ein sich bereits abzeichnendes Ziel ist, die Produktions- und Qualitätsstandards des Automobilsektors 
mit den Fähigkeiten der IT-Konzerne zu vereinen und dadurch einen neuen Standard für alle möglichen automatisierten mobilen Anwendungen zu schaffen. Die Sicherheit wird hierfür als wesentlicher Faktor gesehen und als eine mögliche USP - „automotive safety“ - auf Fachkonferenzen der Technologieentwickler genannt (vgl. Kopetz 2020).

Ein auf aktive Sicherheit ausgelegtes Verkehrssystem, in dem Fahrzeuge umfassend auf historische und aktuelle Daten zurückgreifen können, könnte so aussehen, dass zum Beispiel Kreuzungen, Straßenzüge oder Quartiere, die als nicht sicher gelten, gemieden wären. Wie Gewitterzellen im Flugverkehr würden diese umflogen werden. Ideale Routen führen durch Straßen, die gut vorhersehbar sind. Was für die Autopiloten der Luftfahrt die Physik ist, ist im Straßenraum die Wahrscheinlichkeit, mit der sich das Verhalten anderer Menschen und Objekte im Straßenraum vorhersehen lässt. Der Zugang einer Schule, aus der zweimal täglich größtenteils Personen strömen, die aus dem Vertrauensgrundsatz ausgenommen sind, würde von diesem System zurückhaltend umspielt. Was ist mit Personen oder Personengruppen, die vom Sicherheitssystem als auffällig eingestuft werden?

Abbildung 5: Auswahl aktueller Anwendungen automatisierter und vernetzter Fahrsysteme
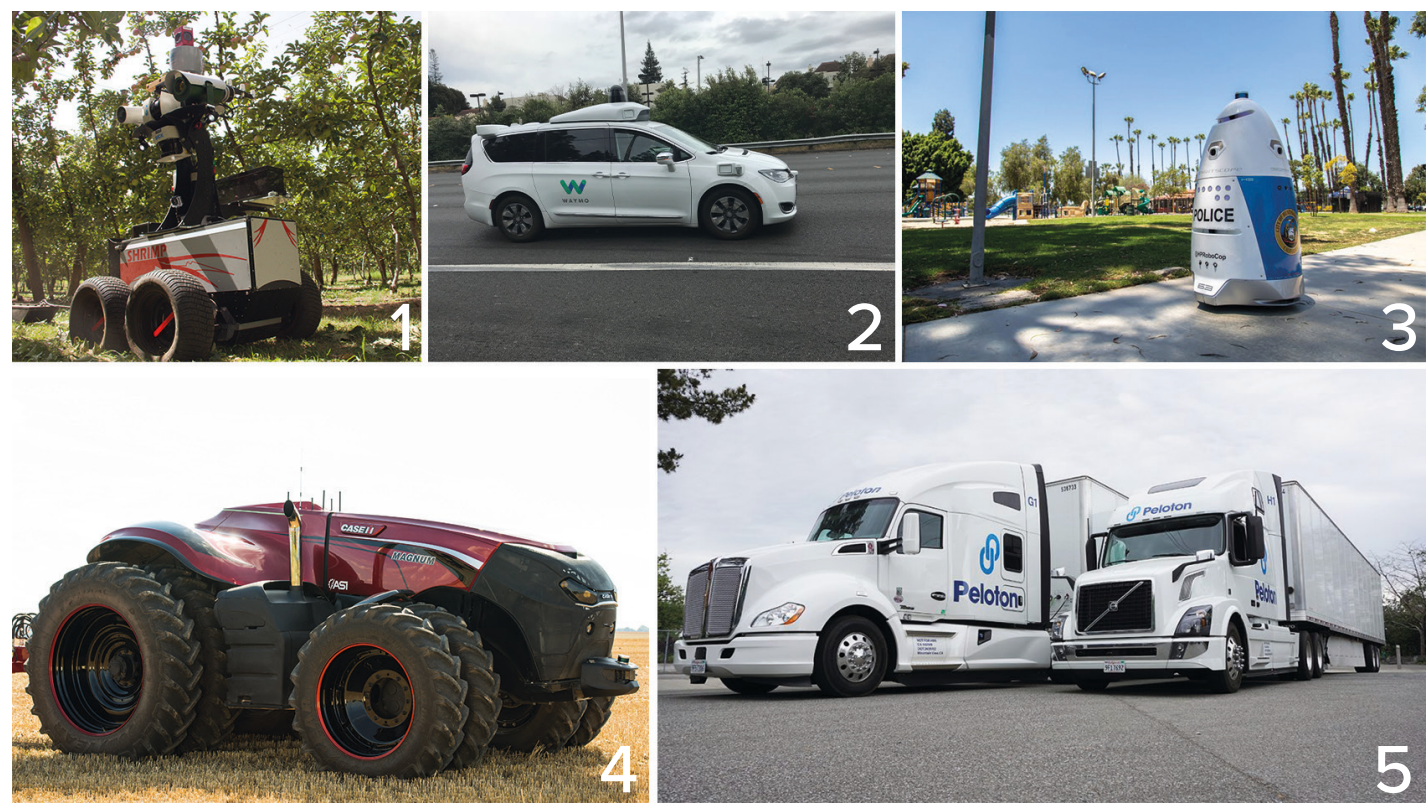

Die hier abgedruckten Bilder sind explizit von der Creative Commons Lizenz des Textes ausgenommen. Die Rechte bleiben bei den Verfassern. Quellen: 1: Australian Centre for Field Robotics (2017), 2: eigenes Foto, 3: knightscope (2021), 4: Casei (2018), 5: peloton-tech o.J.

Paradoxerweise könnte in einem solchen Verkehrssystem mehr Verkehr tatsächlich zu mehr Sicherheit führen. Um bessere Vorhersagen des Verhaltens von Menschen im Straßenraum treffen zu können, müsste die Dichte der Messpunkte im Raum gesteigert werden, sodass das Verhalten besser prognostiziert bzw. manipuliert werden könnte. Geschieht dies nicht durch in der Infrastruktur verbaute Sensorik, könnte dies durch eine Vielzahl von mobilen Robotern geschehen.

Hier ist lange der rein verkehrliche Aspekt der Sicherheit im Straßenraum in den Hintergrund gerückt. Neue Bruchlinien in der Gesellschaft wären durch gezieltes Vermeiden oder durch eine Vielzahl von Sensoren an neuralgischen Punkten (oder Zeiten) unvermeidbar. Diese wären allerdings für Menschen nicht länger verständlich, weil sie in opaken Datensätzen ihren Ursprung nehmen, auf deren Basis künstliches Verhalten geschult wurde (vgl. Castelvecchi 2016). 
Für Sicherheitssysteme, die auf umfassendes Reporting setzten, wurde gezeigt, dass im Zuge der kompletten Aufzeichnung von Daten jeder Vorfall und auch jeder Fast-Vorfall „scheinbar überzeugend" als Problem dargestellt werden kann (Amalberti 2001: 113). Das zugrunde gelegte Verständnis wird schon länger kritisch gesehen: Ein technologisches Konzept verspricht hochtheoretische Leistungs- und Sicherheitspotentiale, menschliches Fehlverhalten wirkt negativ auf die Performance und muss kontrolliert bzw. eliminiert werden. Diese Logik war im Verkehrssektor bislang auf abgegrenzte Bereiche wie Industrie-, Bergbau- oder Logistikarealen bzw. den Flugverkehr und Schienenverkehr beschränkt. Mit automatisierten und vernetzten Fahrsystemen würden diese erstmals den öffentlichen Raum der Straßen betreffen.

Abbildung 6: Veränderte Routenwahl, um unsichere Verkehrssituationen zu vermeiden

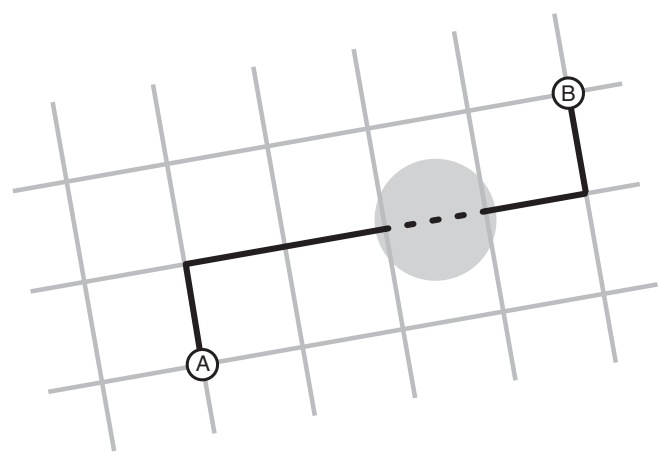

Ein Teilstück der Strecke führt zu unsicheren Verkehrssituationen.

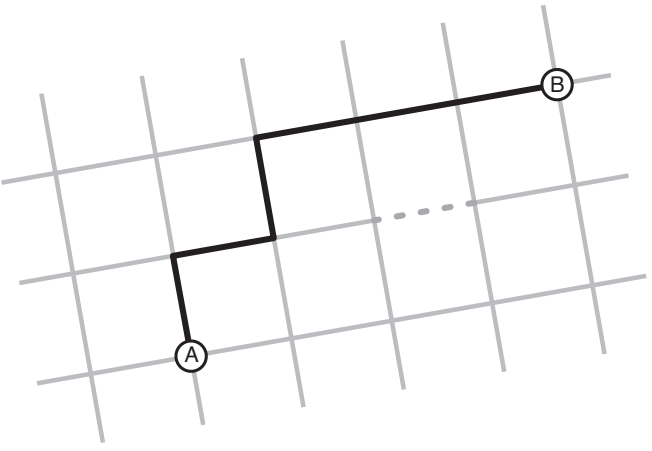

Nach einer Anpassung der Route ist die Strecke durchgehend automatisiert befahrbar.

Quelle: eigene Darstellung nach Alkim in STRIA (2019: 21)

\subsection{EIN NEUES STRASSENMEDIUM}

Die Straße der Moderne, die heute noch den größten Teil unserer bebauten Umwelt ausmacht, ist in vielerlei Hinsicht die perfekte Bühne des dieser Epoche innewohnenden Drangs nach „absoluter Bewegung“ (Jormakka 2002). Die inhärente Spannung, die diesem Paradigma innewohnt - das Verehren von Geschwindigkeit, Rationalität, großen Narrativen und Plänen, während die angestrebte Ordnung durch stetig zunehmende Bewegung immer wieder ins Chaotische abdriftet - hat diese Epoche geprägt. Die Straße des 21. Jahrhunderts könnte unter dem Paradigma „totale Sicherheit“ (Zuboff 2019: 398-415) entstehen. Wie bereits gesagt, handelt es sich hierbei nicht um etwas grundlegend Neues, einen von externen Größen getriebenen Bruch bestehender Pfade, sondern um die exzessive Überhöhung eines bekannten Prinzips. Automatisierte und vernetzte Mobilitätsservices, die situationsabhängige, spontane und flexible Nutzungsformen ermöglichen, forcieren die Zerrissenheit zwischen Chaos und Kontrolle. Der hier aufgezeigte Entwicklungspfad weist nicht in eine Richtung, die darauf abzielt, existentielle Probleme unserer Zeit wie Klimawandel und soziale bzw. Ökonomische Ungleichheit zu lösen. Der Straßenraum dieses neuen Sicherheitssystems würde nicht wie in der Moderne mit Beton gebaut. Kontrolle und Ordnung werden durch Daten und endloses „Nudging“ (Thaler/Sunstein 2009, zur Kritik siehe Stickler/Sodl 2019), dem sanften Beeinflussen des Verhaltens der Personen im Straßenraum, erreicht. Der öffentliche Raum der Straße dieser kuratierten Welt wäre nicht wiederzuerkennen.

Hannah Arendt hat darauf Wert gelegt, dass ein auf Gemeinschaftlichkeit ausgelegtes Zusammenleben der Menschen eine "gemeinsame Welt" braucht, die als eine Basis und Ausgangspunkt einer kritischen Reflexion gesehen werden kann (Arendt 1958: 52-55; Madanipour 2003: 
114-151). Das Gemeinsame können Dinge, Konventionen, eine geteilte Geschichte, gemeinsame Gesetze sind. Es bedarf eines das Zusammenleben strukturierendes „Wir“, das niemals dogmatisch sein kann, sondern als Basis der kritischen Reflexion dient. Wo die Ordnung durch gezielte, personalisierte Verhaltensbeeinflussung hergestellt wird, darf angenommen werden, dass sich diese Basis auflöst. Wer immer die Ziele eines solchen Systems setzt, muss in Kauf nehmen, dass deren Umsetzung undurchsichtig bleibt. Menschen im öffentlichen Raum der Straße würden zu Zwecken werden und die Asymmetrie des generierten Wissens wäre gewaltig (Zuboff 2019).

\section{Öffentlichkeit und Resilienz}

Sich in die Öffentlichkeit zu begeben, bedarf einer „experimentellen Einstellung“, da „Leben Problemlösen ist“ (Gerhardt 2012: 221). Das Ziel totaler Sicherheit, umgesetzt in einem Topdown-Sicherheitssystem im Straßenraum, untergräbt dieses Prinzip in einer bislang nicht bekannten Qualität. Die Automobilität hat bereits an diesem Prinzip gerüttelt. Trotzdem bieten weiterhin Straßen, die als lebendig und divers gelten, Gleichheit, Freiheit und Sicherheit, da darauf vertraut werden kann, dass jedes Verhalten einer kritischen Prüfung der Öffentlichkeit unterworfen wird.

Ähnlich wie in Urrys Szenario können im „Wettbewerb der Städte“, dessen Belohnungssystem das "City Ranking“ ist, die Voraussetzungen geschaffen werden, die die Umsetzung eines solchen Systems begünstigen. Ein kuratiertes Nebeneinander, das bislang vor allem aus Gate Communitys bekannt war, könnte gegenüber dem Gewühl und der Unordnung von Öffentlichkeit in diesen Tabellen sogar besser abschneiden. Die menschliche Entwicklung war seit dem Entstehen sesshafter Kulturen stets an die Entwicklung großer Städte geknüpft. Die stetig zunehmende Komplexität der Siedlung hat die Suche nach Lösungen für die Probleme der Stadt zu einem wichtigen Treiber werden lassen (Hall 1998: 7). Städte mit fortgeschrittenen Volkswirtschaften haben sich weg von der Produktion und dem Warenumschlag und hin zur Dienstleistungsproduktion und weiter zu Informationsgesellschaften entwickelt (Castells 1989, Hall 1995, Hall und Pain 2009). Wir sprechen heute davon, dass Komplexität und Kreativität die zentralen Ressourcen dieser Städte sind. Sie werden von Städten und Regionen unterschieden, die in einer Abhängigkeit zu Rohstoffen stehen, spezialisiert und anfällig für Marktdynamiken sind und meist nur für kurze Zeit florieren. Wo dem öffentlichen Raum sein wandelbarer Charakter genommen wird, und eine externe Instanz umfassend gestaltet, bewertet und verwertet, dort werden auch diese Orte zu einem Spielball externer Kräfte.

\section{LITERATUR}

Adey, P., und D. Bissell 2010. „Mobilities, meetings, and futures: an interview with John Urry”, in Environment and Planning D: Society and Space (28) 1, 1-16.

Alessandrini, A., A. Campagna, P. Delle Site, F. Filippi und L. Persia 2015. „Automated vehicles and the rethinking of mobility and cities“, in Transportation Research Procedia 5, 145-160.

Almeida Correia, G. H. de, D. Milakis, B. van Arem und R. Hoogendoorn 2016. „Vehicle automation and transport system performance“, in: Handbook on Transport and Urban Planning in the Developed World, hg. v. M. C. J. Bliemer, C. Mulley und C. J. Moutou. Cheltenham: Edward Elgar Publishing, 489-516.

Amalberti, R. 2001. „The paradoxes of almost totally safe transportation systems“, in Safety science (37) 2-3, 109-126.

Anderson, P., und M. Tushman 1990. „Technological discontinuities and dominant designs: A cyclical model of technological change“, in Administrative Science Quarterly 35, 604-634. 
Appleyard, D. 1987. „Foreword “, in Public Streets for Public Use, hg. v. A. V. Moudon. New York: Van Nostrand Reinhold, 1-14.

Arendt, H. 1958. The Human Condition. Chicago: The University of Chicago Press.

ASFINAG 2019. „Motorways will start to ,talk' to modern vehicles using WLAN“. https://tinyurl.com/ yclc2whx (3.6.2020).

Australian Centre for Field Robotics (ACFR) 2017. „Our robots“. Mantis and Shrimp. https://confluence. acfr.usyd.edu.au/display/AGPub/Our+Robots (1.2.2021)

Bergek, A., S. Jacobsson, B. Carlsson, S. Lindmark und A. Rickne 2008. „Analyzing the functional dynamics of technological innovation systems: A scheme of analysis“, in Research Policy (37) 3, 407-429.

BMI (Bundesministerium für Inneres) 2020. „Verkehrsstatistik 2019“. Wien. https://tinyurl.com/y9vtdxc2 (3.6.2020).

BMVIT (Bundesministerium für Verkehr, Innovation und Technologie) 2012. „Verkehr in Zahlen. Ausgabe 2011“. Wien. https://tinyurl.com/ycggn5db (4.6.2020).

Boggs, A. M., B. Wali und A. J. Khattak 2020. „Exploratory analysis of automated vehicle crashes in California: a text analytics \& hierarchical Bayesian heterogeneity-based approach“, in Accident Analysis \& Prevention 135, 1-21. DOI: 10.1016/j.aap.2019.105354.

Carreras, A., X. Daura, J. Erhart und S. Ruehrup 2018. „Road infrastructure support levels for automated driving“, Proceedings of the 25th ITS World Congress, 17. -21. September 2018, Kopenhagen, Dänemark. https://tinyurl.com/yb8ffjry (3.6.2020).

Casademont, J., A. Calveras, D. Quiñones, M. Navarro, J. Arribas und M. Catalan-Cid 2019. „Cooperative-Intelligent Transport Systems for Vulnerable Road Users Safety“, in 7th International Conference on Future Internet of Things and Cloud, Istanbul, 141-146. DOI: 10.1109/FiCloud.2019.00027.

Casei 2018. „Athenaeum in Chicago zeichnet autonomen Konzepttraktor als revolutionären Schritt in die Zukunft des Traktordesigns aus“. Presseaussendung. tinyurl.com/4pew59zx (1.2.2021).

Castells, M. 1989. The Informational City: Information Technology, Economic Restructuring and the Urban-Regional Process. Oxford: Basil Blackwell.

Castelvecchi, D. 2016. „Can we open the black box of Al?“, in Nature News (538) 7623, 20.

Cresswell, T. 2006. On the Move: Mobility in the Modern Western World. New York/London: Routledge.

EEA (European Environment Agency) 2009. „Ensuring quality of life in Europe's cities and towns“, EEA Report 5 2009. Kopenhagen: European Environment Agency.

Elvik, R., A. Høye, T. Vaa und M. Sørensen 2009. The Handbook of Road Safety Measures. Bingley, UK: Emerald Group Publishing Limited.

ERSO (European Road Safety Observatory) 2019. „Annual Accident Report 2018“. https://tinyurl.com/ y94vaeo4 (29.5.2020).

European Commission 2017a. „C-ITS platform PHASE II - Final Report“, Brüssel. https://tinyurl.com/ y7hzgxt9 (4.6.2020).

European Commission 2017b. „Connected and Automated Transport. Studies and reports“. Brüssel: European Commission, Directorate-General for Research and Innovation. Download unter: https:// tinyurl.com/y89radja (4.6.2020).

Feigenbaum, B., G. Goodin, A. Kim, S. Kimmel, R. Mudge und D. Perlman 2018. „Policymaking for Automated Vehicles: A Proactive Approach for Government", in Road Vehicle Automation 4, hg. v. G. Meyer und S. Beiker. Cham: Springer, 33-41.

Foucault, M. 1981. Archäologie des Wissens. Frankfurt am Main: Suhrkamp.

Foucault, M. 2007. Security, Territory, Population: Lectures at the Collège de France 1977-1978. London: Palgrave Macmillan.

Foucault, M. 2012. Überwachen und Strafen: Die Geburt des Gefängnisses. Frankfurt am Main: Suhrkamp.

Fustel de Coulanges, N. D. 1979. The ancient city: a study on the religion, laws, and institutions of Greece and Rome. Gloucester, MA: Peter Smith.

Geels, F. W., und J. Schot 2007. „Typology of sociotechnical transition pathways“, in Research Policy (36) 3, 399-417.

Gehl, I. 2009. Public Spaces \& Public Life. Seattle: City of Seattle. 
Georgieva, T., und K. Kolodege 2018. „Bridging the Automated Vehicle Gap: Consumer Trust, Technology and Liability“, Vortrag beim Automated Vehicle Symposium 2018, 10. Juli 2018. https://tinyurl. com/yba9tx9s (29.5.2020).

Gerhardt, V. 2012. Öffentlichkeit. München: C. H. Beck.

Gopnik, A. 2016. „Street Cred“, The New Yorker (92) 30.

Gratz, R. B. 2010. The Battle for Gotham: New York in the Shadow of Robert Moses and Jane Jacobs. New York: Bold Type Books.

Grübler, A. 1992. „Technology and Global Change: Land Use, Past and Present“, IIASA Working Paper WP-92-002, Laxenburg.

Hall, P. G. 1995. „Towards a General Urban Theory“, in Cities in Competition: Productive and Sustainable Cities for the 21st Century, hg. v. J. Brotchie, M. Batty, E. Blakely, P. Hall und P. Newton. Melbourne: Longman Australia, 3-31.

Hall, P. G. 1998. Cities in Civilization. London: Weidenfeld \& Nicolson.

Hall, P., und K. Pain 2009. The Polycentric Metropolis: Learning from Mega-City Regions in Europe. London: Routledge.

Hodder, I., und P. Pels 2010. „History houses: a new interpretation of architectural elaboration at Çatalhöyük", in Religion in the Emergence of Civilization: Çatalhöyük as a Case Study, hg. v. I. Hodder. Cambridge, UK: Cambridge University Press, 163-186.

ITF (International Transport Forum) 2015. „Urban Mobility System Upgrade. How shared self-driving cars could change city traffic“, Corporate Partnership Board Report. Paris: ITC. https://tinyurl.com/ yaeylous (29.5.2020).

Jacobs, J. 1961. The Death and Life of Great American Cities. New York: Random House.

Jing, P., G. Xu, Y. Chen, Y. Shi und F. Zhan 2020. „The Determinants behind the Acceptance of Autonomous Vehicles: A Systematic Review“, in Sustainability (12) 5, 1719, 1-16.

Jormakka, K. (Hrsg.) 2002. „Absolute Motion“, Datutop 22. Tampere, Fl: Tampere University of Technology.

Kang, Y., H. Yin und C. Berger 2019. „Test your self-driving algorithm: An overview of publicly available driving datasets and virtual testing environments", in IEEE Transactions on Intelligent Vehicles (4) 2, 171-185.

Kopetz, Hermann 2020. „An Architecture for Driving Automation“. https://www.the-autonomous.com/ news/an-architecture-for-driving-automation/ (24.8.2020).

knightscope 2021. „Knightscope Credited for Reducing Crime“. Blogbeitrag. https://www.knightscope. com/blog-1/2021/01/07/crime/ (1.2.2021).

KPMG 2012. „Self-driving cars: The next revolution“. https://tinyurl.com/y6woqoks (29.5.2020).

Landini, S. 2020. „Ethical Issues, Cybersecurity and Automated Vehicles“, in InsurTech: A Legal and Regulatory View, hg. v. P. Marano und K. Noussia. Cham: Springer, 291-312.

Lazarus, J., S. Shaheen, S. E. Young, D. Fagnant, T. Voege, W. Baumgardner und J. S. Lott 2018. „Shared automated mobility and public transport", in Road Vehicle Automation 4, hg. v. G. Meyer und S. Beiker. Cham: Springer, 141-161.

Lenz, B., und E. Fraedrich 2015. „Neue Mobilitätskonzepte und autonomes Fahren: Potenziale der Veränderung“, in Autonomes Fahren. Technische, rechtliche und gesellschaftliche Aspekte, hg. v. M. Maurer, J. C. Gerdes, B. Lenz und H. Winner. Berlin/Heidelberg: Springer Vieweg, 175-196.

Madanipour, A. 2003. Public and Private Spaces of the City. London/New York: Routledge.

Maia, S. C., und A. Meyboom 2018. „Understanding the effects of autonomous vehicles on urban form“, in Road Vehicle Automation 4, hg. v. G. Meyer und S. Beiker. Cham: Springer, 201-221.

Marchetti, C. 1994. „Anthropological Invariants in Travel Behavior“, in Technological Forecasting and Social Change 47, 75-88.

Marshall, S. 2009. Cities, Design \& Evolution. New York: Routledge.

Marx, P. 2018. „Self-Driving Cars Are Out. Micromobility Is In“, Medium, 15.11.2018. https://tinyurl.com/ y7g88778 (3.6.2020).

Massey, D., 2005. For Space. London: Sage Publications. 
McLuhan, M. 1960. Report on Project in Understanding New Media. Washington: National Association of Educational Broadcasters.

McShane, C. 1994. Down the Asphalt Path: The Automobile and the American City. New York: Columbia University Press.

Meyer, G. 2019. „European roadmaps, programs, and projects for innovation in connected and automated road transport", in Road Vehicle Automation 5, hg. v. G. Meyer und S. Beiker. Cham: Springer, 27-39.

Mitteregger, M. 2020. „Pandemien beschleunigen den Ersatz von Menschen durch Maschinen“, Wiener Zeitung, 21.4.2020. https://tinyurl.com/y7k42vma (3.6.2020).

Mitteregger, M., A. Soteropoulos, J. Bröthaler und F. Dorner 2019. „Shared, Automated, Electric: the Fiscal Effects of the ,Holy Trinity“", in Proceedings of the 24. REAL CORP, International Conference on Urban Planning, Regional Development and Information Society, 627-636. https://tinyurl.com/ yamvccho (3.6.2020).

Mitteregger, M., M. Berger und A. Soteropoulos 2020a. „Algorithmen von morgen, die in einer Welt von gestern lernen: Das Inselwissen automatisierter Fahrsysteme“, in Mobilität - Erreichbarkeit - Ländliche Räume ... und die Frage nach der Gleichwertigkeit der Lebensverhältnisse, hg. v. M. Herget, S. Neumeier und T. Osigus. Braunschweig: Thünen-Institut für Ländliche Räume, 75-77.

Mitteregger, M., E. M. Bruck, A. Soteropoulos, A. Stickler, M. Berger, J. S. Dangschat, R. Scheuvens und I. Banerjee 2020b. AVENUE21. Automatisierter und vernetzter Verkehr: Entwicklungen des urbanen Europa. Berlin: Springer Vieweg.

Newman, P., L. Kosonen und J. Kenworthy 2016. „Theory of urban fabrics: Planning the walking, transit/ public transport and automobile/motor car cities for reduced car dependency“, in Town Planning Review (87) 4, 429-458.

NHTSA (National Highway Traffic Safety Administration) 2017. „Automated Driving Systems 2.0: A Vision for Safety“. https://tinyurl.com/y87nzq3q (3.6.2020).

peloton-tech o. J. https://peloton-tech.com/wp-content/uploads/2017/04/Peloton1-1024x576.jpg (1.2.2021).

POLIS (European Cities and Regions Networking for Innovative Transport Solutions) 2018. „Road Vehicle Automation and Cities and Regions“. Brüssel: Polis. https://tinyurl.com/yb8ppy9h (3.6.2020).

Reki, B. 2004. Kultur als Praxis. Eine Einführung in Ernst Cassierers Philosophie der symbolischen Formen. Berlin: Akademie Verlag.

Rogers, E. M. 2003. Diffusion of innovations. New York: Free Press.

Rykwert, J. 1986. „The street: The use of its history“, in On Streets, hg. v. S. Anderson. Cambridge, MA: MIT Press, 14-27.

Sartre, J. 1962. Das Sein und das Nichts. Reinbek: Rowohlt.

Schoettle, B. 2017. „Sensor Fusion: A Comparison of Sensing Capabilities of Human Drivers and Highly Automated Vehicles“, Report No. SWT-2017-12. Ann Arbor, MA: University of Michigan, Transportation Research Institute.

Schumpeter, J. A. 1939. Business Cycles. A Theoretical, Historical and Statistical Analysis of the Capitalist Process. New York/Toronto/London: McGraw-Hill.

Seo, S. A. (2019). Policing the Open Road: How Cars Transformed American Freedom. Cambridge, MA: Harvard University Press.

Shakland, S. 2016. „Al expert: Super-smart cars are just a glorious beginning“, CNET, 21.10.2016. https:// tinyurl.com/jv7yo8y (3.6.2020).

Sheller, M., und J. Urry 2006. „The new mobilities paradigm“, in Environment and Planning A: Economy and Space (38) 2, 207-226.

Shladover, S. E., J. Lappin und R. P. Denaro 2019. „Introduction: The Automated Vehicles Symposium 2017“, in Road Vehicle Automation 5, hg. v. G. Meyer und S. Beiker. Cham: Springer, 1-14.

Simmel, G. 1903. „Die Grosstädte und das Geistesleben“, in Die Grossstadt. Vorträge und Aufsätze zur Städteausstellung, Jahrbuch der Gehe-Stiftung Dresden, hg. v. T. Petermann, Band 9, 185-206.

Simmel, G. 1908. Soziologie. Untersuchungen über die Formen der Vergesellschaftung, 1. Aufl. Berlin: Duncker \& Humblot.

Soteropoulos, A., M. Mitteregger, M. Berger und J. Zwirchmayr 2020. „Automated drivability: Toward an assessment of the spatial deployment of level 4 automated vehicles“, in Transportation Research Part A: Policy and Practice 136, 64-84. 
Statistik Austria 2017. „Straßenverkehrsunfälle mit Personenschaden. Jahresergebnisse 2016“. https:// tinyurl.com/y8qakyxv (3.6.2020).

Stickler, A., und V. Sodl 2019. „Nudging als Ansatz zur Förderung von nachhaltiger Mobilität? Potentiale und Risiken von verkehrspsychologischen Ansätzen zur ökologischen Nachhaltigkeitstransformation“, in Jahrbuch Raumplanung 2019, hg. v. M. Berger, J. Forster, M. Getzner und P. Hirschler. Wien: NWV Verlag, 75-96.

STRIA 2019. „Roadmap on Connected and Automated Transport: Road, Rail and Waterborne“. Brüssel: Europäische Kommission.

Thaler, R. H., und C. R. Sunstein 2009. Nudge: Improving decisions about health, wealth, and happiness. München: Penguin.

Urry, J. 2007. Mobilities. London: Polity.

Urry, J. 2008. „Climate change, travel and complex futures“, in The British Journal of Sociology (59) 2 , 261-279.

Vrkljan, B. H., und D. Anaby 2011. „What vehicle features are considered important when buying an automobile? An examination of driver preferences by age and gender", in Journal of safety research (42) 1, 61-65.

Winkle, T. (2015). „Sicherheitspotenzial automatisierter Fahrzeuge: Erkenntnisse aus der Unfallforschung", in Autonomes Fahren. Technische, rechtliche und gesellschaftliche Aspekte, hg. v. M. Maurer, J. C. Gerdes, B. Lenz und H. Winner. Berlin/Heidelberg: Springer Vieweg, 351-376.

WHO (World Health Organization) 2015. „Global Status Report on Road Safety 2015“. Genf: World Health Organization.

Zuboff, S. 2019. The age of surveillance capitalism: The fight for a human future at the new frontier of power. London: Profile Books.

Open Access Dieser Beitrag wird unter der Creative Commons Namensnennung 4.0 International Lizenz (http://creativecommons.org/licenses/by/4.0/deed.de) veröffentlicht, welche die Nutzung, Vervielfältigung, Bearbeitung, Verbreitung und Wiedergabe in jeglichem Medium und Format erlaubt, sofern Sie den/die ursprünglichen Autor(en) und die Quelle ordnungsgemäß nennen, einen Link zur Creative Commons Lizenz beifügen und angeben, ob Änderungen vorgenommen wurden.

Die in diesem Beitrag enthaltenen Bilder und sonstiges Drittmaterial unterliegen ebenfalls der genannten Creative Commons Lizenz, sofern sich aus der Abbildungslegende nichts anderes ergibt. Sofern das betreffende Material nicht unter der genannten Creative Commons Lizenz steht und die betreffende Handlung nicht nach gesetzlichen Vorschriften erlaubt ist, ist für die oben aufgeführten Weiterverwendungen des Materials die Einwilligung des jeweiligen Rechteinhabers einzuholen.

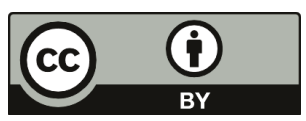

